\title{
SOME VARIANTS OF THE SZEGED INDEX UNDER ROOTED PRODUCT OF GRAPHS
}

\author{
MAHDIEH AZARI \\ Received 10 November, 2015
}

\begin{abstract}
The Szeged index $S z(G)$ of a connected graph $G$ is defined as the sum of the terms $n_{u}(e \mid G) n_{v}(e \mid G)$ over all edges $e=u v$ of $G$, where $n_{u}(e \mid G)$ is the number of vertices of $G$ lying closer to $u$ than to $v$ and $n_{v}(e \mid G)$ is the number of vertices of $G$ lying closer to $v$ than to $u$. In this paper, some variants of the Szeged index such as the edge PI index, edge Szeged index, edge-vertex Szeged index, vertex-edge Szeged index, and revised edge Szeged index are studied under rooted product of graphs. Results are applied to compute these graph invariants for some chemical graphs by specializing components in rooted products.
\end{abstract}

2010 Mathematics Subject Classification: 05C12;05C76

Keywords: edge PI index, edge Szeged index, edge-vertex Szeged index, vertex-edge Szeged index, revised edge Szeged index, rooted product of graphs

\section{INTRODUCTION}

In this paper, we consider connected finite graphs without loops or multiple edges. Let $G$ be such a graph with vertex set $V(G)$ and edge set $E(G)$. We denote by $d(u, v \mid G)$ the distance between the vertices $u$ and $v$ in $G$ which is the length of any shortest path in $G$ connecting $u$ and $v$. Let $e=u v$ be the edge of $G$ connecting the vertices $u$ and $v$. The quantities $n_{0}(e \mid G), n_{u}(e \mid G)$, and $n_{v}(e \mid G)$ are defined to be the number of vertices of $G$ equidistant from $u$ and $v$, the number of vertices of $G$ whose distance to $u$ is smaller than the distance to $v$, and the number of vertices of $G$ whose distance to $v$ is smaller than the distance to $u$, respectively, i.e.,

$$
\begin{aligned}
& n_{0}(e \mid G)=|\{z \in V(G): d(z, u \mid G)=d(z, v \mid G)\}|, \\
& n_{u}(e \mid G)=|\{z \in V(G): d(z, u \mid G)<d(z, v \mid G)\}|, \\
& n_{v}(e \mid G)=|\{z \in V(G): d(z, v \mid G)<d(z, u \mid G)\}| .
\end{aligned}
$$

For an edge $e=u v \in E(G)$ and a vertex $z \in V(G)$, the distance between $z$ and $e$ is defined as $d(z, e \mid G)=\min \{d(z, u \mid G), d(z, v \mid G)\}$. The quantities $m_{0}(e \mid G)$, $m_{u}(e \mid G)$, and $m_{v}(e \mid G)$ are defined to be the number of edges of $G$ equidistant from $u$ and $v$, the number of edges of $G$ whose distance to $u$ is smaller than the distance 
to $v$, and the number of edges of $G$ whose distance to $v$ is smaller than the distance to $u$, respectively, i.e.,

$$
\begin{aligned}
& m_{0}(e \mid G)=|\{f \in E(G): d(u, f \mid G)=d(v, f \mid G)\}|, \\
& m_{u}(e \mid G)=|\{f \in E(G): d(u, f \mid G)<d(v, f \mid G)\}|, \\
& m_{v}(e \mid G)=|\{f \in E(G): d(v, f \mid G)<d(u, f \mid G)\}| .
\end{aligned}
$$

For the vertex $z \in V(G)$, we define

$$
m_{z}(G)=|\{e=u v \in E(G): d(u, z \mid G) \neq d(v, z \mid G)\}| .
$$

Chemical graphs, particularly molecular graphs, are graph-based descriptions of molecules, with vertices representing the atoms and edges representing the bonds. A numerical invariant associated with a chemical graph is called topological index or graph invariant. Topological indices are used in theoretical chemistry for the design of chemical compounds with given physicochemical properties or given pharmacologic and biological activities [6,20]. The Wiener index [21], defined as the sum of distances between all pairs of vertices in a chemical graph, is the oldest and the most thoroughly studied topological index from both theoretical and practical point of view. Motivated by the original definition of the Wiener index, the Szeged index [11] was introduced in 1994 which coincides with the Wiener index for a tree. It found applications in quantitative structure-property-activity-toxicity modeling [16]. The Szeged index of a graph $G$ is defined as

$$
S z(G)=\sum_{e=u v \in E(G)} n_{u}(e \mid G) n_{v}(e \mid G) .
$$

In recent years, some variants of the Szeged index such as the vertex PI index [17], edge PI index [15], edge Szeged index [12], edge-vertex Szeged index [18], vertexedge Szeged index [9], revised Szeged index [19], and revised edge Szeged index [7] have attracted much attention in both chemistry and mathematics. These indices are defined for a graph $G$ as follows:

$$
\begin{aligned}
P I_{v}(G) & =\sum_{e=u v \in E(G)}\left[n_{u}(e \mid G)+n_{v}(e \mid G)\right], \\
P I_{e}(G) & =\sum_{e=u v \in E(G)}\left[m_{u}(e \mid G)+m_{v}(e \mid G)\right], \\
S z_{e}(G) & =\sum_{e=u v \in E(G)} m_{u}(e \mid G) m_{v}(e \mid G), \\
S z_{e v}(G) & =\frac{1}{2} \sum_{e=u v \in E(G)}\left[n_{u}(e \mid G) m_{v}(e \mid G)+n_{v}(e \mid G) m_{u}(e \mid G)\right],
\end{aligned}
$$




$$
\begin{aligned}
S z_{v e}(G) & =\frac{1}{2} \sum_{e=u v \in E(G)}\left[n_{u}(e \mid G) m_{u}(e \mid G)+n_{v}(e \mid G) m_{v}(e \mid G)\right], \\
S z^{*}(G) & =\sum_{e=u v \in E(G)}\left[n_{u}(e \mid G)+\frac{n_{0}(e \mid G)}{2}\right]\left[n_{v}(e \mid G)+\frac{n_{0}(e \mid G)}{2}\right], \\
S z_{e}^{*}(G) & =\sum_{e=u v \in E(G)}\left[m_{u}(e \mid G)+\frac{m_{0}(e \mid G)}{2}\right]\left[m_{v}(e \mid G)+\frac{m_{0}(e \mid G)}{2}\right] .
\end{aligned}
$$

We refer the reader to $[1,10,14]$ for more information on these indices.

The rooted product $G_{1}\left\{G_{2}\right\}$ of a graph $G_{1}$ and a rooted graph $G_{2}$ is the graph obtained by taking one copy of $G_{1}$ and $\left|V\left(G_{1}\right)\right|$ copies of $G_{2}$, and by identifying the root vertex of the $i$-th copy of $G_{2}$ with the $i$-th vertex of $G_{1}$, for $i=1,2, \ldots,\left|V\left(G_{1}\right)\right|$.

In this paper, we study the edge PI index, edge Szeged index, edge-vertex Szeged index, vertex-edge Szeged index, and revised edge Szeged index under rooted product of graphs. Results are applied to compute these invariants for some chemical graphs by specializing components in rooted products. For more information on computing topological indices of rooted product see $[2-5,8,13,22]$.

\section{RESULTS AND DISCUSSION}

Let $G_{1}$ and $G_{2}$ be two connected graphs with vertex sets $V\left(G_{1}\right)$ and $V\left(G_{2}\right)$ and edge sets $E\left(G_{1}\right)$ and $E\left(G_{2}\right)$, respectively. In this section, we compute some variants of the Szeged index for rooted product of $G_{1}$ and $G_{2}$. Throughout this section, the graph $G_{2}$ is assumed to be rooted on the vertex $x \in V\left(G_{2}\right)$ and the degree of $x$ in $G_{2}$ is denoted by $\delta$. Also, we denote by $n_{i}$ and $m_{i}$, the order and size of the graph $G_{i}$, respectively, where $i \in\{1,2\}$. In addition, for notational convenience, we define

$$
\bar{N}=\sum_{\substack{e=u v \in E\left(G_{2}\right), d\left(u, x \mid G_{2}\right)<d\left(v, x \mid G_{2}\right)}} n_{v}\left(e \mid G_{2}\right), \quad \underline{N}=\sum_{\begin{array}{c}
e=u v \in E\left(G_{2}\right), \\
\left(u, x \mid G_{2}\right)<d\left(v, x \mid G_{2}\right) \\
e=u v \in E\left(G_{2}\right), \\
\left(u, x \mid G_{2}\right)<d\left(v, x \mid G_{2}\right)
\end{array}} m_{v}\left(e \mid G_{2}\right), \quad \underline{M}=\sum_{\begin{array}{c}
e=u v \in E\left(G_{2}\right), \\
d\left(u, x \mid G_{2}\right)<d\left(v, x \mid G_{2}\right)
\end{array}} m_{u}\left(e \mid G_{2}\right) .
$$

Theorem 1. The edge PI index of the rooted product $G_{1}\left\{G_{2}\right\}$ is given by

$$
\begin{aligned}
P I_{e}\left(G_{1}\left\{G_{2}\right\}\right)= & P I_{e}\left(G_{1}\right)+m_{2} P I_{v}\left(G_{1}\right)+n_{1} P I_{e}\left(G_{2}\right) \\
& +n_{1}\left(m_{1}+\left(n_{1}-1\right) m_{2}\right) m_{x}\left(G_{2}\right) .
\end{aligned}
$$

Proof. From the definition of the edge PI index, we have

$$
P I_{e}\left(G_{1}\left\{G_{2}\right\}\right)=\sum_{e=u v \in E\left(G_{1}\left\{G_{2}\right\}\right)}\left[m_{u}\left(e \mid G_{1}\left\{G_{2}\right\}\right)+m_{v}\left(e \mid G_{1}\left\{G_{2}\right\}\right)\right] .
$$

We partition the above sum into two sums as follows: 
The first sum $S_{1}$ consists of contributions to $P I_{e}\left(G_{1}\left\{G_{2}\right\}\right)$ of edges from $G_{1}$,

$$
S_{1}=\sum_{e=u v \in E\left(G_{1}\right)}\left[m_{u}\left(e \mid G_{1}\left\{G_{2}\right\}\right)+m_{v}\left(e \mid G_{1}\left\{G_{2}\right\}\right)\right] .
$$

By definition of rooted product, we have

$$
\begin{aligned}
S_{1}= & \sum_{e=u v \in E\left(G_{1}\right)}\left[\left(m_{u}\left(e \mid G_{1}\right)+m_{2} n_{u}\left(e \mid G_{1}\right)\right)+\left(m_{v}\left(e \mid G_{1}\right)+m_{2} n_{v}\left(e \mid G_{1}\right)\right)\right] \\
= & \sum_{e=u v \in E\left(G_{1}\right)}\left[m_{u}\left(e \mid G_{1}\right)+m_{v}\left(e \mid G_{1}\right)\right] \\
& +m_{2} \sum_{e=u v \in E\left(G_{1}\right)}\left[n_{u}\left(e \mid G_{1}\right)+n_{v}\left(e \mid G_{1}\right)\right] \\
= & P I_{e}\left(G_{1}\right)+m_{2} P I_{v}\left(G_{1}\right) .
\end{aligned}
$$

The second sum $S_{2}$ consists of contributions to $P I_{e}\left(G_{1}\left\{G_{2}\right\}\right)$ of edges from $n_{1}$ copies of $G_{2}$,

$$
S_{2}=n_{1} \sum_{e=u v \in E\left(G_{2}\right)}\left[m_{u}\left(e \mid G_{1}\left\{G_{2}\right\}\right)+m_{v}\left(e \mid G_{1}\left\{G_{2}\right\}\right)\right] .
$$

By definition of rooted product, we have

$$
\begin{aligned}
S_{2}= & n_{1} \sum_{\begin{array}{r}
e=u v \in E\left(G_{2}\right), \\
d\left(u, x \mid G_{2}\right) \neq d\left(v, x \mid G_{2}\right)
\end{array}}\left[m_{1}+\left(n_{1}-1\right) m_{2}+m_{u}\left(e \mid G_{2}\right)+m_{v}\left(e \mid G_{2}\right)\right] \\
+ & n_{1} \sum_{\begin{array}{r}
e=u v \in E\left(G_{2}\right), \\
d\left(u, x \mid G_{2}\right)=d\left(v, x \mid G_{2}\right)
\end{array}}\left[m_{u}\left(e \mid G_{2}\right)+m_{v}\left(e \mid G_{2}\right)\right] \\
= & n_{1}\left[\sum_{\begin{array}{c}
e=u v \in E\left(G_{2}\right), \\
d\left(u, x \mid G_{2}\right) \neq d\left(v, x \mid G_{2}\right)
\end{array}}\left[m_{u}\left(e \mid G_{2}\right)+m_{v}\left(e \mid G_{2}\right)\right]\right. \\
& \left.+\sum_{\begin{array}{c}
e=u v \in E\left(G_{2}\right), \\
d\left(u, x \mid G_{2}\right)=d\left(v, x \mid G_{2}\right)
\end{array}}\left[m_{u}\left(e \mid G_{2}\right)+m_{v}\left(e \mid G_{2}\right)\right]\right] \\
& +n_{1}\left(m_{1}+\left(n_{1}-1\right) m_{2}\right)\left|\left\{e=u v \in E\left(G_{2}\right): d\left(u, x \mid G_{2}\right) \neq d\left(v, x \mid G_{2}\right)\right\}\right| \\
= & n_{1} P I_{e}\left(G_{2}\right)+n_{1}\left(m_{1}+\left(n_{1}-1\right) m_{2}\right) m_{x}\left(G_{2}\right) .
\end{aligned}
$$

Eq. (2.1) is obtained by adding the quantities $S_{1}$ and $S_{2}$.

Theorem 2. The edge Szeged index of the rooted product $G_{1}\left\{G_{2}\right\}$ is given by

$$
\begin{aligned}
S z_{e}\left(G_{1}\left\{G_{2}\right\}\right)= & S z_{e}\left(G_{1}\right)+m_{2}^{2} S z\left(G_{1}\right)+2 m_{2} S z_{e v}\left(G_{1}\right)+n_{1} S z_{e}\left(G_{2}\right) \\
& +n_{1}\left(m_{1}+\left(n_{1}-1\right) m_{2}\right) \bar{M} .
\end{aligned}
$$


Proof. From the definition of the edge Szeged index, we have

$$
S z_{e}\left(G_{1}\left\{G_{2}\right\}\right)=\sum_{e=u v \in E\left(G_{1}\left\{G_{2}\right\}\right)} m_{u}\left(e \mid G_{1}\left\{G_{2}\right\}\right) m_{v}\left(e \mid G_{1}\left\{G_{2}\right\}\right) .
$$

We partition the above sum into two sums as follows:

The first sum $S_{1}$ consists of contributions to $S z_{e}\left(G_{1}\left\{G_{2}\right\}\right)$ of edges from $G_{1}$,

$$
S_{1}=\sum_{e=u v \in E\left(G_{1}\right)} m_{u}\left(e \mid G_{1}\left\{G_{2}\right\}\right) m_{v}\left(e \mid G_{1}\left\{G_{2}\right\}\right) .
$$

By definition of rooted product, we have

$$
\begin{aligned}
S_{1}= & \sum_{e=u v \in E\left(G_{1}\right)}\left[m_{u}\left(e \mid G_{1}\right)+m_{2} n_{u}\left(e \mid G_{1}\right)\right]\left[m_{v}\left(e \mid G_{1}\right)+m_{2} n_{v}\left(e \mid G_{1}\right)\right] \\
= & \sum_{e=u v \in E\left(G_{1}\right)} m_{u}\left(e \mid G_{1}\right) m_{v}\left(e \mid G_{1}\right)+m_{2}^{2} \sum_{e=u v \in E\left(G_{1}\right)} n_{u}\left(e \mid G_{1}\right) n_{v}\left(e \mid G_{1}\right) \\
& +m_{2} \sum_{e=u v \in E\left(G_{1}\right)}\left[n_{u}\left(e \mid G_{1}\right) m_{v}\left(e \mid G_{1}\right)+n_{v}\left(e \mid G_{1}\right) m_{u}\left(e \mid G_{1}\right)\right] \\
= & S z_{e}\left(G_{1}\right)+m_{2}^{2} S z\left(G_{1}\right)+2 m_{2} S z_{e v}\left(G_{1}\right) .
\end{aligned}
$$

The second sum $S_{2}$ consists of contributions to $S z_{e}\left(G_{1}\left\{G_{2}\right\}\right)$ of edges from $n_{1}$ copies of $G_{2}$,

$$
S_{2}=n_{1} \sum_{e=u v \in E\left(G_{2}\right)} m_{u}\left(e \mid G_{1}\left\{G_{2}\right\}\right) m_{v}\left(e \mid G_{1}\left\{G_{2}\right\}\right) .
$$

By definition of rooted product, we have

$$
\begin{aligned}
& S_{2}=n_{1} \sum_{\begin{array}{c}
e=u v \in E\left(G_{2}\right), \\
d\left(u, x \mid G_{2}\right)<d\left(v, x \mid G_{2}\right)
\end{array}}\left[m_{1}+\left(n_{1}-1\right) m_{2}+m_{u}\left(e \mid G_{2}\right)\right] m_{v}\left(e \mid G_{2}\right) \\
& +n_{1} \sum_{e=u v \in E\left(G_{2}\right),} m_{u}\left(e \mid G_{2}\right) m_{v}\left(e \mid G_{2}\right) \\
& d\left(u, x \mid G_{2}\right)=d\left(v, x \mid G_{2}\right) \\
& =n_{1}\left[\sum_{\substack{e=u v \in E\left(G_{2}\right), d\left(u, x \mid G_{2}\right)<d\left(v, x \mid G_{2}\right)}} m_{u}\left(e \mid G_{2}\right) m_{v}\left(e \mid G_{2}\right)\right. \\
& \left.+\sum_{\begin{array}{c}
e=u v \in E\left(G_{2}\right), \\
d\left(u, x \mid G_{2}\right)=d\left(v, x \mid G_{2}\right)
\end{array}} m_{u}\left(e \mid G_{2}\right) m_{v}\left(e \mid G_{2}\right)\right] \\
& +n_{1}\left(m_{1}+\left(n_{1}-1\right) m_{2}\right) \sum_{e=u v \in E\left(G_{2}\right)} m_{v}\left(e \mid G_{2}\right) \\
& d\left(u, x \mid G_{2}\right)<d\left(v, x \mid G_{2}\right) \\
& =n_{1} S z_{e}\left(G_{2}\right)+n_{1}\left(m_{1}+\left(n_{1}-1\right) m_{2}\right) \bar{M} \text {. }
\end{aligned}
$$


Eq. (2.2) is obtained by adding the quantities $S_{1}$ and $S_{2}$.

Theorem 3. The edge-vertex Szeged index of the rooted product $G_{1}\left\{G_{2}\right\}$ is given by

$$
\begin{aligned}
S z_{e v}\left(G_{1}\left\{G_{2}\right\}\right)= & n_{2} S z_{e v}\left(G_{1}\right)+n_{2} m_{2} S z\left(G_{1}\right)+n_{1} S z_{e v}\left(G_{2}\right)+\frac{1}{2} n_{1} n_{2}\left(n_{1}-1\right) \bar{M} \\
& +\frac{1}{2} n_{1}\left(m_{1}+m_{2}\left(n_{1}-1\right)\right) \bar{N} .
\end{aligned}
$$

Proof. From the definition of the edge-vertex Szeged index, we have

$$
\begin{aligned}
S z_{e v}\left(G_{1}\left\{G_{2}\right\}\right)= & \frac{1}{2} \sum_{e=u v \in E\left(G_{1}\left\{G_{2}\right\}\right)}\left[n_{u}\left(e \mid G_{1}\left\{G_{2}\right\}\right) m_{v}\left(e \mid G_{1}\left\{G_{2}\right\}\right)\right. \\
& \left.+n_{v}\left(e \mid G_{1}\left\{G_{2}\right\}\right) m_{u}\left(e \mid G_{1}\left\{G_{2}\right\}\right)\right] .
\end{aligned}
$$

We partition the above sum into two sums as follows:

The first sum $S_{1}$ consists of contributions to $S z_{e v}\left(G_{1}\left\{G_{2}\right\}\right)$ of edges from $G_{1}$,

$S_{1}=\frac{1}{2} \sum_{e=u v \in E\left(G_{1}\right)}\left[n_{u}\left(e \mid G_{1}\left\{G_{2}\right\}\right) m_{v}\left(e \mid G_{1}\left\{G_{2}\right\}\right)+n_{v}\left(e \mid G_{1}\left\{G_{2}\right\}\right) m_{u}\left(e \mid G_{1}\left\{G_{2}\right\}\right)\right]$.

By definition of rooted product, we have

$$
\begin{aligned}
S_{1}= & \frac{1}{2} \sum_{e=u v \in E\left(G_{1}\right)}\left[n_{2} n_{u}\left(e \mid G_{1}\right)\left[m_{v}\left(e \mid G_{1}\right)+m_{2} n_{v}\left(e \mid G_{1}\right)\right]\right. \\
& \left.+n_{2} n_{v}\left(e \mid G_{1}\right)\left[m_{u}\left(e \mid G_{1}\right)+m_{2} n_{u}\left(e \mid G_{1}\right)\right]\right] \\
= & \frac{1}{2} n_{2} \sum_{e=u v \in E\left(G_{1}\right)}\left[n_{u}\left(e \mid G_{1}\right) m_{v}\left(e \mid G_{1}\right)+n_{v}\left(e \mid G_{1}\right) m_{u}\left(e \mid G_{1}\right)\right] \\
& +n_{2} m_{2} \sum_{e=u v \in E\left(G_{1}\right)} n_{u}\left(e \mid G_{1}\right) n_{v}\left(e \mid G_{1}\right) \\
= & n_{2} S z_{e v}\left(G_{1}\right)+n_{2} m_{2} S z\left(G_{1}\right) .
\end{aligned}
$$

The second sum $S_{2}$ consists of contributions to $S z_{e v}\left(G_{1}\left\{G_{2}\right\}\right)$ of edges from $n_{1}$ copies of $G_{2}$,

$$
\begin{aligned}
S_{2}= & \frac{1}{2} n_{1} \sum_{e=u v \in E\left(G_{2}\right)}\left[n_{u}\left(e \mid G_{1}\left\{G_{2}\right\}\right) m_{v}\left(e \mid G_{1}\left\{G_{2}\right\}\right)\right. \\
& \left.+n_{v}\left(e \mid G_{1}\left\{G_{2}\right\}\right) m_{u}\left(e \mid G_{1}\left\{G_{2}\right\}\right)\right] .
\end{aligned}
$$

By definition of rooted product, we have

$$
S_{2}=\frac{1}{2} n_{1} \sum_{\begin{array}{c}
e=u v \in E\left(G_{2}\right), \\
d\left(u, x \mid G_{2}\right)<d\left(v, x \mid G_{2}\right)
\end{array}}\left[\left[n_{u}\left(e \mid G_{2}\right)+n_{2}\left(n_{1}-1\right)\right] m_{v}\left(e \mid G_{2}\right)\right.
$$




$$
\begin{aligned}
& \left.+n_{v}\left(e \mid G_{2}\right)\left[m_{1}+m_{2}\left(n_{1}-1\right)+m_{u}\left(e \mid G_{2}\right)\right]\right] \\
& +\frac{1}{2} n_{1} \sum_{e=u v \in E\left(G_{2}\right),}\left[n_{u}\left(e \mid G_{2}\right) m_{v}\left(e \mid G_{2}\right)+n_{v}\left(e \mid G_{2}\right) m_{u}\left(e \mid G_{2}\right)\right] \\
& \begin{array}{c}
e=u v \in E\left(G_{2}\right), \\
d\left(u, x \mid G_{2}\right)=d\left(v, x \mid G_{2}\right)
\end{array} \\
& =\frac{1}{2} n_{1}\left[\sum_{\substack{e=u v \in E\left(G_{2}\right), d\left(u, x \mid G_{2}\right)<d\left(v, x \mid G_{2}\right)}}\left[n_{u}\left(e \mid G_{2}\right) m_{v}\left(e \mid G_{2}\right)+n_{v}\left(e \mid G_{2}\right) m_{u}\left(e \mid G_{2}\right)\right]\right. \\
& \left.+\sum_{\begin{array}{c}
e=u v \in E\left(G_{2}\right), \\
d\left(u, x \mid G_{2}\right)=d\left(v, x \mid G_{2}\right)
\end{array}}\left[n_{u}\left(e \mid G_{2}\right) m_{v}\left(e \mid G_{2}\right)+n_{v}\left(e \mid G_{2}\right) m_{u}\left(e \mid G_{2}\right)\right]\right] \\
& +\frac{1}{2} n_{1} n_{2}\left(n_{1}-1\right) \sum_{\substack{e=u v \in E\left(G_{2}\right), d\left(u, x \mid G_{2}\right)<d\left(v, x \mid G_{2}\right)}} m_{v}\left(e \mid G_{2}\right) \\
& +\frac{1}{2} n_{1}\left(m_{1}+m_{2}\left(n_{1}-1\right)\right) \sum_{e=u v \in E\left(G_{2}\right),} n_{v}\left(e \mid G_{2}\right) \\
& d\left(u, x \mid G_{2}\right)<d\left(v, x \mid G_{2}\right) \\
& =n_{1} S z_{e v}\left(G_{2}\right)+\frac{1}{2} n_{1} n_{2}\left(n_{1}-1\right) \bar{M}+\frac{1}{2} n_{1}\left(m_{1}+m_{2}\left(n_{1}-1\right)\right) \bar{N} .
\end{aligned}
$$

Eq. (2.3) is obtained by adding the quantities $S_{1}$ and $S_{2}$.

Let $G$ be a graph with edge set $E(G)$. We define the second vertex PI index of $G$ as

$$
P I_{v}^{(2)}(G)=\sum_{e=u v \in E(G)}\left[n_{u}(e \mid G)^{2}+n_{v}(e \mid G)^{2}\right] .
$$

Theorem 4 ( [9]). Let $G$ be a graph of order $n$ and size $m$. Then

$$
S z^{*}(G)=\frac{m n^{2}}{4}-\frac{1}{4} P I_{v}^{(2)}(G)+\frac{1}{2} S z(G) .
$$

Theorem 5. The vertex-edge Szeged index of the rooted product $G_{1}\left\{G_{2}\right\}$ is given by

$$
\begin{aligned}
S z_{v e}\left(G_{1}\left\{G_{2}\right\}\right)= & n_{2} S z_{v e}\left(G_{1}\right)+\frac{1}{2} n_{2} m_{2} P I_{v}^{(2)}\left(G_{1}\right)+n_{1} S z_{v e}\left(G_{2}\right) \\
& +\frac{1}{2} n_{1} n_{2}\left(n_{1}-1\right)\left(m_{1}+m_{2}\left(n_{1}-1\right)\right) m_{x}\left(G_{2}\right) \\
& +\frac{1}{2} n_{1}\left(m_{1}+m_{2}\left(n_{1}-1\right)\right) \underline{N}+\frac{1}{2} n_{1} n_{2}\left(n_{1}-1\right) \underline{M} .
\end{aligned}
$$


Proof. From the definition of the vertex-edge Szeged index, we have

$$
\begin{aligned}
S z_{v e}\left(G_{1}\left\{G_{2}\right\}\right)= & \frac{1}{2} \sum_{e=u v \in E\left(G_{1}\left\{G_{2}\right\}\right)}\left[n_{u}\left(e \mid G_{1}\left\{G_{2}\right\}\right) m_{u}\left(e \mid G_{1}\left\{G_{2}\right\}\right)\right. \\
& \left.+n_{v}\left(e \mid G_{1}\left\{G_{2}\right\}\right) m_{v}\left(e \mid G_{1}\left\{G_{2}\right\}\right)\right] .
\end{aligned}
$$

We partition the above sum into two sums as follows:

The first sum $S_{1}$ consists of contributions to $S z_{v e}\left(G_{1}\left\{G_{2}\right\}\right)$ of edges from $G_{1}$,

$$
S_{1}=\frac{1}{2} \sum_{e=u v \in E\left(G_{1}\right)}\left[n_{u}\left(e \mid G_{1}\left\{G_{2}\right\}\right) m_{u}\left(e \mid G_{1}\left\{G_{2}\right\}\right)+n_{v}\left(e \mid G_{1}\left\{G_{2}\right\}\right) m_{v}\left(e \mid G_{1}\left\{G_{2}\right\}\right)\right] .
$$

By definition of rooted product, we have

$$
\begin{aligned}
S_{1}= & \frac{1}{2} \sum_{e=u v \in E\left(G_{1}\right)}\left[n_{2} n_{u}\left(e \mid G_{1}\right)\left[m_{u}\left(e \mid G_{1}\right)+m_{2} n_{u}\left(e \mid G_{1}\right)\right]\right. \\
& \left.+n_{2} n_{v}\left(e \mid G_{1}\right)\left[m_{v}\left(e \mid G_{1}\right)+m_{2} n_{v}\left(e \mid G_{1}\right)\right]\right] \\
= & \frac{1}{2} n_{2} \sum_{e=u v \in E\left(G_{1}\right)}\left[n_{u}\left(e \mid G_{1}\right) m_{u}\left(e \mid G_{1}\right)+n_{v}\left(e \mid G_{1}\right) m_{v}\left(e \mid G_{1}\right)\right] \\
& +\frac{1}{2} n_{2} m_{2} \sum_{e=u v \in E\left(G_{1}\right)}\left[n_{u}\left(e \mid G_{1}\right)^{2}+n_{v}\left(e \mid G_{1}\right)^{2}\right] \\
= & n_{2} S z_{v e}\left(G_{1}\right)+\frac{1}{2} n_{2} m_{2} P I_{v}^{(2)}\left(G_{1}\right) .
\end{aligned}
$$

The second sum $S_{2}$ consists of contributions to $S z_{v e}\left(G_{1}\left\{G_{2}\right\}\right)$ of edges from $n_{1}$ copies of $G_{2}$,

$$
\begin{aligned}
S_{2}= & \frac{1}{2} n_{1} \sum_{e=u v \in E\left(G_{2}\right)}\left[n_{u}\left(e \mid G_{1}\left\{G_{2}\right\}\right) m_{u}\left(e \mid G_{1}\left\{G_{2}\right\}\right)\right. \\
& \left.+n_{v}\left(e \mid G_{1}\left\{G_{2}\right\}\right) m_{v}\left(e \mid G_{1}\left\{G_{2}\right\}\right)\right] .
\end{aligned}
$$

By definition of rooted product, we have

$$
\begin{aligned}
S_{2}= & \frac{1}{2} n_{1} \sum_{\begin{array}{c}
e=u v \in E\left(G_{2}\right), \\
d\left(u, x \mid G_{2}\right)<d\left(v, x \mid G_{2}\right)
\end{array}}\left[[ n _ { u } ( e | G _ { 2 } ) + n _ { 2 } ( n _ { 1 } - 1 ) ] \left[m_{1}+m_{2}\left(n_{1}-1\right)\right.\right. \\
& \left.\left.+m_{u}\left(e \mid G_{2}\right)\right]+n_{v}\left(e \mid G_{2}\right) m_{v}\left(e \mid G_{2}\right)\right] \\
& +\frac{1}{2} n_{1} \sum_{\begin{array}{c}
e=u v \in E\left(G_{2}\right), \\
d\left(u, x \mid G_{2}\right)=d\left(v, x \mid G_{2}\right)
\end{array}}\left[n_{u}\left(e \mid G_{2}\right) m_{u}\left(e \mid G_{2}\right)+n_{v}\left(e \mid G_{2}\right) m_{v}\left(e \mid G_{2}\right)\right]
\end{aligned}
$$




$$
\begin{aligned}
= & \frac{1}{2} n_{1}\left[\sum_{\begin{array}{c}
e=u v \in E\left(G_{2}\right), \\
d\left(u, x \mid G_{2}\right)<d\left(v, x \mid G_{2}\right)
\end{array}}\left[n_{u}\left(e \mid G_{2}\right) m_{u}\left(e \mid G_{2}\right)+n_{v}\left(e \mid G_{2}\right) m_{v}\left(e \mid G_{2}\right)\right]\right. \\
& \left.+\sum_{\substack{e=u v \in E\left(G_{2}\right), d\left(u, x \mid G_{2}\right)=d\left(v, x \mid G_{2}\right)}}\left[n_{u}\left(e \mid G_{2}\right) m_{u}\left(e \mid G_{2}\right)+n_{v}\left(e \mid G_{2}\right) m_{v}\left(e \mid G_{2}\right)\right]\right] \\
& +\frac{1}{2} n_{1} n_{2}\left(n_{1}-1\right)\left(m_{1}+m_{2}\left(n_{1}-1\right)\right) \mid\left\{e=u v \in E\left(G_{2}\right): d\left(u, x \mid G_{2}\right)\right. \\
< & \left.d\left(v, x \mid G_{2}\right)\right\} \mid+\frac{1}{2} n_{1}\left(m_{1}+m_{2}\left(n_{1}-1\right)\right) \sum_{\substack{e=u v \in E\left(G_{2}\right), d\left(u, x \mid G_{2}\right)<d\left(v, x \mid G_{2}\right)}} n_{u}\left(e \mid G_{2}\right) \\
& +\frac{1}{2} n_{1} n_{2}\left(n_{1}-1\right) \quad \sum_{\substack{e=u v \in E\left(G_{2}\right), d\left(u, x \mid G_{2}\right)<d\left(v, x \mid G_{2}\right)}} m_{u}\left(e \mid G_{2}\right) \\
= & n_{1} S z_{v e}\left(G_{2}\right)+\frac{1}{2} n_{1} n_{2}\left(n_{1}-1\right)\left(m_{1}+m_{2}\left(n_{1}-1\right)\right) m_{x}\left(G_{2}\right) \\
& +\frac{1}{2} n_{1}\left(m_{1}+m_{2}\left(n_{1}-1\right)\right) \underline{N}+\frac{1}{2} n_{1} n_{2}\left(n_{1}-1\right) \underline{M} .
\end{aligned}
$$

Eq. (2.5) is obtained by adding the quantities $S_{1}$ and $S_{2}$.

Using Eq. (2.4), we can get an alternative formula for the vertex-edge Szeged index of the rooted product of $G_{1}$ and $G_{2}$.

Corollary 1. The vertex-edge Szeged index of the rooted product $G_{1}\left\{G_{2}\right\}$ is given by

$$
\begin{aligned}
S z_{v e}\left(G_{1}\left\{G_{2}\right\}\right)= & n_{2} S z_{v e}\left(G_{1}\right)+n_{2} m_{2} S z\left(G_{1}\right)-2 n_{2} m_{2} S z^{*}\left(G_{1}\right)+n_{1} S z_{v e}\left(G_{2}\right) \\
& +\frac{1}{2} n_{1}^{2} n_{2} m_{1} m_{2}+\frac{1}{2} n_{1} n_{2}\left(n_{1}-1\right)\left(m_{1}+m_{2}\left(n_{1}-1\right)\right) m_{x}\left(G_{2}\right) \\
& +\frac{1}{2} n_{1} n_{2}\left(n_{1}-1\right) \underline{M}+\frac{1}{2} n_{1}\left(m_{1}+m_{2}\left(n_{1}-1\right)\right) \underline{N} .
\end{aligned}
$$

Proof. From Eq. (2.4), we get

$$
P I_{v}^{(2)}\left(G_{1}\right)=m_{1} n_{1}^{2}-4 S z^{*}\left(G_{1}\right)+2 S z\left(G_{1}\right) .
$$

Eq. (2.6) is obtained by applying the above equation in Eq. (2.5) and simplifying the resulting expression.

Let $G$ be a graph with edge set $E(G)$. We define the second edge PI index of $G$ as

$$
P I_{e}^{(2)}(G)=\sum_{e=u v \in E(G)}\left[m_{u}(e \mid G)^{2}+m_{v}(e \mid G)^{2}\right] .
$$


Theorem 6 ([9]). Let $G$ be a graph of size $m$. Then

$$
S z_{e}^{*}(G)=\frac{m^{3}}{4}-\frac{1}{4} P I_{e}^{(2)}(G)+\frac{1}{2} S z_{e}(G)
$$

Lemma 1. The second edge PI index of $G_{1}\left\{G_{2}\right\}$ is given by

$$
\begin{aligned}
P I_{e}^{(2)}\left(G_{1}\left\{G_{2}\right\}\right)= & P I_{e}^{(2)}\left(G_{1}\right)+m_{2}^{2} P I_{v}^{(2)}\left(G_{1}\right)+4 m_{2} S z_{v e}\left(G_{1}\right) \\
& +n_{1} P I_{e}^{(2)}\left(G_{2}\right)+n_{1}\left(m_{1}+\left(n_{1}-1\right) m_{2}\right)^{2} m_{x}\left(G_{2}\right) \\
& +2 n_{1}\left(m_{1}+\left(n_{1}-1\right) m_{2}\right) \underline{M} .
\end{aligned}
$$

Proof. From the definition of the second edge PI index, we have

$$
P I_{e}^{(2)}\left(G_{1}\left\{G_{2}\right\}\right)=\sum_{e=u v \in E\left(G_{1}\left\{G_{2}\right\}\right)}\left[m_{u}\left(e \mid G_{1}\left\{G_{2}\right\}\right)^{2}+m_{v}\left(e \mid G_{1}\left\{G_{2}\right\}\right)^{2}\right] .
$$

We partition the above sum into two sums as follows:

The first sum $S_{1}$ consists of contributions to $P I_{e}^{(2)}\left(G_{1}\left\{G_{2}\right\}\right)$ of edges from $G_{1}$,

$$
S_{1}=\sum_{e=u v \in E\left(G_{1}\right)}\left[m_{u}\left(e \mid G_{1}\left\{G_{2}\right\}\right)^{2}+m_{v}\left(e \mid G_{1}\left\{G_{2}\right\}\right)^{2}\right] .
$$

By definition of rooted product, we have

$$
\begin{aligned}
S_{1}= & \sum_{e=u v \in E\left(G_{1}\right)}\left[\left(m_{u}\left(e \mid G_{1}\right)+m_{2} n_{u}\left(e \mid G_{1}\right)\right)^{2}+\left(m_{v}\left(e \mid G_{1}\right)+m_{2} n_{v}\left(e \mid G_{1}\right)\right)^{2}\right] \\
= & \sum_{e=u v \in E\left(G_{1}\right)}\left[m_{u}\left(e \mid G_{1}\right)^{2}+m_{v}\left(e \mid G_{1}\right)^{2}\right] \\
& +m_{2}^{2} \sum_{e=u v \in E\left(G_{1}\right)}\left[n_{u}\left(e \mid G_{1}\right)^{2}+n_{v}\left(e \mid G_{1}\right)^{2}\right] \\
& +2 m_{2} \sum_{e=u v \in E\left(G_{1}\right)}\left[n_{u}\left(e \mid G_{1}\right) m_{u}\left(e \mid G_{1}\right)+n_{v}\left(e \mid G_{1}\right) m_{v}\left(e \mid G_{1}\right)\right] \\
= & P I_{e}^{(2)}\left(G_{1}\right)+m_{2}^{2} P I_{v}^{(2)}\left(G_{1}\right)+4 m_{2} S z_{v e}\left(G_{1}\right) .
\end{aligned}
$$

The second sum $S_{2}$ consists of contributions to $P I_{e}^{(2)}\left(G_{1}\left\{G_{2}\right\}\right)$ of edges from $n_{1}$ copies of $G_{2}$,

$$
S_{2}=n_{1} \sum_{e=u v \in E\left(G_{2}\right)}\left[m_{u}\left(e \mid G_{1}\left\{G_{2}\right\}\right)^{2}+m_{v}\left(e \mid G_{1}\left\{G_{2}\right\}\right)^{2}\right] .
$$

By definition of rooted product, we have

$$
S_{2}=n_{1} \sum_{\begin{array}{c}
e=u v \in E\left(G_{2}\right), \\
d\left(u, x \mid G_{2}\right)<d\left(v, x \mid G_{2}\right)
\end{array}}\left[\left(m_{1}+\left(n_{1}-1\right) m_{2}+m_{u}\left(e \mid G_{2}\right)\right)^{2}+m_{v}\left(e \mid G_{2}\right)^{2}\right]
$$




$$
\begin{aligned}
& +n_{1} \sum_{e=u v \in E\left(G_{2}\right),}\left[m_{u}\left(e \mid G_{2}\right)^{2}+m_{v}\left(e \mid G_{2}\right)^{2}\right] \\
& d\left(u, x \mid G_{2}\right)=d\left(v, x \mid G_{2}\right) \\
& =n_{1}\left[\sum_{\substack{e=u v \in E\left(G_{2}\right), d\left(u, x \mid G_{2}\right)<d\left(v, x \mid G_{2}\right)}}\left[m_{u}\left(e \mid G_{2}\right)^{2}+m_{v}\left(e \mid G_{2}\right)^{2}\right]\right. \\
& \left.+\sum_{\begin{array}{c}
e=u v \in E\left(G_{2}\right), \\
d\left(u, x \mid G_{2}\right)=d\left(v, x \mid G_{2}\right)
\end{array}}\left[m_{u}\left(e \mid G_{2}\right)^{2}+m_{v}\left(e \mid G_{2}\right)^{2}\right]\right] \\
& +n_{1}\left(m_{1}+\left(n_{1}-1\right) m_{2}\right)^{2}\left|\left\{e=u v \in E\left(G_{2}\right): d\left(u, x \mid G_{2}\right)<d\left(v, x \mid G_{2}\right)\right\}\right| \\
& +2 n_{1}\left(m_{1}+\left(n_{1}-1\right) m_{2}\right) \sum_{e=u v \in E\left(G_{2}\right),} m_{u}\left(e \mid G_{2}\right) \\
& d\left(u, x \mid G_{2}\right)<d\left(v, x \mid G_{2}\right) \\
& =n_{1} P I_{e}^{(2)}\left(G_{2}\right)+n_{1}\left(m_{1}+\left(n_{1}-1\right) m_{2}\right)^{2} m_{x}\left(G_{2}\right)+2 n_{1}\left(m_{1}+\left(n_{1}-1\right) m_{2}\right) \underline{M} .
\end{aligned}
$$

Eq. (2.8) is obtained by adding the quantities $S_{1}$ and $S_{2}$.

Theorem 7. The revised edge Szeged index of the rooted product $G_{1}\left\{G_{2}\right\}$ is given by

$$
\begin{aligned}
S z_{e}^{*}\left(G_{1}\left\{G_{2}\right\}\right)= & S z_{e}^{*}\left(G_{1}\right)+m_{2}^{2} S z^{*}\left(G_{1}\right)+m_{2}\left(S z_{e v}\left(G_{1}\right)-S z_{v e}\left(G_{1}\right)\right) \\
& +n_{1} S z_{e}^{*}\left(G_{2}\right)+\frac{1}{2} n_{1}\left(m_{1}+\left(n_{1}-1\right) m_{2}\right)(\bar{M}-\underline{M}) \\
& -\frac{1}{4} n_{1}\left(m_{1}+\left(n_{1}-1\right) m_{2}\right)^{2} m_{x}\left(G_{2}\right) \\
& +\frac{1}{4} n_{1} m_{2}\left[m_{1}\left(3 m_{1}+2 n_{1} m_{2}\right)+m_{2}^{2}\left(n_{1}^{2}-1\right)\right] .
\end{aligned}
$$

Proof. By Eq. (2.7),

$$
S z_{e}^{*}\left(G_{1}\left\{G_{2}\right\}\right)=\frac{1}{4}\left(m_{1}+n_{1} m_{2}\right)^{3}-\frac{1}{4} P I_{e}^{(2)}\left(G_{1}\left\{G_{2}\right\}\right)+\frac{1}{2} S z_{e}\left(G_{1}\left\{G_{2}\right\}\right) .
$$

Now using Eq. (2.2) and Eq. (2.8), we get

$$
\begin{aligned}
S z_{e}^{*}\left(G_{1}\left\{G_{2}\right\}\right)= & \frac{1}{4}\left[m_{1}^{3}+3 m_{1}^{2} n_{1} m_{2}+3 m_{1} n_{1}^{2} m_{2}^{2}+n_{1}^{3} m_{2}^{3}\right]-\frac{1}{4}\left[P I_{e}^{(2)}\left(G_{1}\right)\right. \\
& +m_{2}^{2} P I_{v}^{(2)}\left(G_{1}\right)+4 m_{2} S z_{v e}\left(G_{1}\right)+n_{1} P I_{e}^{(2)}\left(G_{2}\right) \\
& \left.+n_{1}\left(m_{1}+\left(n_{1}-1\right) m_{2}\right)^{2} m_{x}\left(G_{2}\right)+2 n_{1}\left(m_{1}+\left(n_{1}-1\right) m_{2}\right) \underline{M}\right] \\
& +\frac{1}{2}\left[S z_{e}\left(G_{1}\right)+m_{2}^{2} S z\left(G_{1}\right)+2 m_{2} S z_{e v}\left(G_{1}\right)+n_{1} S z_{e}\left(G_{2}\right)\right. \\
& \left.+n_{1}\left(m_{1}+\left(n_{1}-1\right) m_{2}\right) \bar{M}\right] .
\end{aligned}
$$

Eq. (2.9) is obtained by simplifying the above expression. 


\section{EXAMPLES AND COROLLARIES}

In this section, we apply the results of the previous section to compute the edge PI index, edge Szeged index, edge-vertex Szeged index, vertex-edge Szeged index, and revised edge Szeged index of some graphs by specializing components in rooted product.

Let $P_{n}, S_{n}$, and $C_{n}$ denote the $n$-vertex path, star, and cycle, respectively. Some Szeged-related topological indices of these graphs have been given in Table 1.

\begin{tabular}{r|r|r|r|r} 
Graph & $P_{n}$ & $S_{n}$ & $C_{n}, n$ is even & $C_{n}, n$ is odd \\
\hline$P I_{v}$ & $n(n-1)$ & $n(n-1)$ & $n^{2}$ & $n(n-1)$ \\
$P I_{e}$ & $(n-1)(n-2)$ & $(n-1)(n-2)$ & $n(n-2)$ & $n(n-1)$ \\
$S z$ & $\left(\begin{array}{c}n+1 \\
3\end{array}\right)$ & $(n-1)^{2}$ & $\frac{n^{3}}{4}$ & $\frac{n(n-1)^{2}}{4}$ \\
$S z_{e}$ & $\left(\begin{array}{c}n-1 \\
3\end{array}\right)$ & 0 & $\frac{n(n-2)^{2}}{4}$ & $\frac{n(n-1)^{2}}{4}$ \\
$S z_{e v}$ & $\left(\begin{array}{c}n \\
3\end{array}\right)$ & $\frac{(n-1)(n-2)}{2}$ & $\frac{n^{2}(n-2)}{2}$ & $\frac{n(n-1)^{2}}{2}$ \\
$S z_{v e}$ & $2\left(\begin{array}{c}n \\
3\end{array}\right)$ & $\frac{(n-1)^{2}(n-2)}{2}$ & $\frac{n^{2}(n-2)}{2}$ & $\frac{n(n-1)^{2}}{2}$ \\
$S z^{*}$ & $\left(\begin{array}{c}n+1 \\
3\end{array}\right)$ & $(n-1)^{2}$ & $\frac{n^{3}}{4}$ & $\frac{n^{3}}{4}$ \\
$S z_{e}^{*}$ & $\frac{(n-1)\left(2 n^{2}-4 n+3\right)}{12}$ & $\frac{(n-1)(2 n-3)}{4}$ & $\frac{n^{3}}{4}$ & $\frac{n^{3}}{4}$ \\
\hline \hline
\end{tabular}

TABLE 1. Some topological indices of path, star, and cycle.

As the first example, consider the rooted product of $P_{n}$ and $P_{m}$, where the root vertex of $P_{m}$ is assumed to be on one of its pendant vertices (vertices of degree one). This molecular graph is called the comb lattice graph. Using Eqs. (2.1)-(2.3), (2.6), (2.9), and Table 1, we easily arrive at:

Corollary 2. Let $G=P_{n}\left\{P_{m}\right\}$, where the root vertex of $P_{m}$ is assumed to be on one of its pendant vertices. Then

(i) $P I_{e}(G)=(n m-1)(n m-2)$,

(ii) $S z_{e}(G)=\frac{n m^{3}}{6}(3 n-2)+\frac{n m^{2}}{6}\left(n^{2}-9 n+2\right)+\frac{11 n m}{6}-1$,

(iii) $S z_{e v}(G)=\frac{n m^{3}}{6}(3 n-2)+\frac{n m^{2}}{6}\left(n^{2}-6 n+2\right)+\frac{n m}{3}$,

(iv) $S z_{v e}(G)=\frac{n m^{3}}{6}\left(3 n^{2}-3 n+2\right)-\frac{n m^{2}}{6}(n+1)(n+2)+\frac{2 n m}{3}$,

(v) $S z_{e}^{*}(G)=\frac{n m^{3}}{6}(3 n-2)+\frac{n m^{2}}{6}\left(n^{2}-6 n+2\right)+\frac{7 n m}{12}-\frac{1}{4}$.

Let $P_{n}^{*}(m)$ denote the $m$-thorn path which is the graph obtained by attaching $m$ pendant vertices to each vertex of the path $P_{n}$. This graph can be viewed as the rooted 
product of $P_{n}$ and the star graph on $m+1$ vertices, where the root vertex of $S_{m+1}$ is assumed to be on its central vertex (vertex of degree $m$ ). Using Eqs. (2.1)-(2.3), (2.6), (2.9), and Table 1, we easily arrive at:

Corollary 3. The following equalities hold:

(i) $P I_{e}\left(P_{n}^{*}(m)\right)=n^{2} m^{2}+n m(2 n-3)+(n-1)(n-2)$,

(ii) $S z_{e}\left(P_{n}^{*}(m)\right)=\frac{n m^{2}}{6}(n-1)(n+1)+\frac{n m}{3}(n-1)(n-2)+\left(\begin{array}{c}n-1 \\ 3\end{array}\right)$,

(iii) $S z_{e v}\left(P_{n}^{*}(m)\right)=\frac{n m^{2}}{6}\left(n^{2}+3 n-1\right)+\frac{n m}{6}\left(2 n^{2}-5\right)+\left(\begin{array}{l}n \\ 3\end{array}\right)$,

(iv) $S z_{v e}\left(P_{n}^{*}(m)\right)=\frac{n^{3} m^{3}}{2}+\frac{n m^{2}}{6}\left(8 n^{2}-12 n+1\right)+\frac{n m}{6}(n-1)(7 n-11)$

$$
+2\left(\begin{array}{l}
n \\
3
\end{array}\right)
$$

(v) $S z_{e}^{*}\left(P_{n}^{*}(m)\right)=\frac{n m^{2}}{6}\left(n^{2}+3 n-1\right)+\frac{n m}{12}\left(4 n^{2}-7\right)+\frac{n}{12}\left(2 n^{2}-6 n+7\right)-\frac{1}{4}$.

Let $C_{n}^{*}(m)$ denote the $m$-thorn cycle which is the graph obtained by attaching $m$ pendant vertices to each vertex of the cycle $C_{n}$. This graph can be seen as the rooted product of $C_{n}$ and the star graph on $m+1$ vertices, where $S_{m+1}$ is assumed to be rooted on its central vertex. Using Eqs. (2.1)-(2.3), (2.6), (2.9), and Table 1, we easily arrive at:

Corollary 4. The following equalities hold:

(i) $P I_{e}\left(C_{n}^{*}(m)\right)=\left\{\begin{array}{l}n^{2} m^{2}+n m(2 n-1)+n(n-2) \\ n^{2} m^{2}+2 n m(n-1)+n(n-1)\end{array}\right.$

$n$ is even,

(ii) $S z_{e}\left(C_{n}^{*}(m)\right)=\left\{\begin{array}{l}\frac{n(n m+n-2)^{2}}{4} \\ \frac{n(n-1)^{2}(m+1)^{2}}{4}\end{array}\right.$ $n$ is odd,

$n$ is even,

$n$ is odd,

(iii) $S z_{e v}\left(C_{n}^{*}(m)\right)= \begin{cases}\frac{n^{2} m^{2}}{4}(n+2)+\frac{n m}{2}\left(n^{2}-1\right)+\frac{n^{2}}{4}(n-2) & n \text { is even, } \\ \frac{n m^{2}}{4}\left(n^{2}+1\right)+\frac{n^{2} m}{2}(n-1)+\frac{n(n-1)^{2}}{4} & n \text { is odd, }\end{cases}$

(iv) $S z_{v e}\left(C_{n}^{*}(m)\right)= \begin{cases}\frac{n^{3} m^{3}}{2}+\frac{n^{2} m^{2}}{4}(5 n-4) & \text { nis even } \\ +\frac{n m}{2}\left(2 n^{2}-3 n+1\right)+\frac{n^{2}(n-2)}{4}, & \\ \frac{n^{3} m^{3}}{2}+\frac{n m^{2}}{4}\left(5 n^{2}-6 n+1\right) & n \text { is odd } \\ +n m(n-1)^{2}+\frac{n(n-1)^{2}}{4}, & \end{cases}$

(v) $S z_{e}^{*}\left(C_{n}^{*}(m)\right)=\frac{n^{2} m^{2}}{4}(n+2)+\frac{n m}{4}\left(2 n^{2}+2 n-1\right)+\frac{n^{3}}{4}$. 
Finally, consider the rooted product of $P_{n}$ and $C_{m}$. Note that because of the symmetry of $C_{m}$ any vertex of this graph can be considered at its root vertex. Using Eqs. (2.1)-(2.3), (2.6), (2.9), and Table 1, we easily arrive at:

Corollary 5. Let $G=P_{n}\left\{C_{m}\right\}$. Then

(i) $P I_{e}(G)=\left\{\begin{array}{l}n^{2} m^{2}+2 n m(n-2)+(n-1)(n-2) \\ n^{2} m^{2}+n m(n-2)-2(n-1)\end{array}\right.$ $m$ is even,

(ii) $S z_{e}(G)= \begin{cases}\frac{n m^{3}}{4}(2 n-1)+\frac{n m^{2}}{6}(n+1)(n-4) & \text { mis eve } \\ +\frac{n m}{3}(n-2)(n-4)+\left(\begin{array}{c}n-1 \\ 3\end{array}\right), & \\ \frac{n m^{3}}{4}(2 n-1)+\frac{n m^{2}}{6}\left(n^{2}-3 n-1\right) & \text { mis odd } \\ +\frac{n m}{12}\left(4 n^{2}-18 n+17\right)+\frac{n-1}{6}\left(n^{2}-2 n+6\right), & \end{cases}$

(iii) $S z_{\text {ev }}(G)=\left\{\begin{array}{lr}\frac{n^{2} m^{3}}{2}+\frac{n m^{2}}{12}\left(2 n^{2}-3 n-11\right)+m\left(\begin{array}{c}n \\ 3\end{array}\right) & \text { mis even, } \\ \frac{n^{2} m^{3}}{2}+\frac{n m^{2}}{12}\left(2 n^{2}-9 n-5\right) & \text { mis odd } \\ +\frac{n m}{6}\left(n^{2}-3 n+5\right)+\frac{n(n-1)}{4}, & \end{array}\right.$

(iv) $S z_{v e}(G)= \begin{cases}\frac{n m^{3}}{2}\left(n^{2}-n+1\right)+\frac{n m^{2}}{12}\left(10 n^{2}-21 n-1\right) & \text { mis even } \\ +2 m\left(\begin{array}{l}n \\ 3\end{array}\right), & \\ \frac{n m^{3}}{2}\left(n^{2}-n+1\right)+\frac{n m^{2}}{12}\left(4 n^{2}-15 n-1\right) & \text { m is odd } \\ -\frac{n m}{6}\left(n^{2}-4\right)+\frac{n(n-1)}{4}, & \end{cases}$

(v) $S z_{e}^{*}(G)= \begin{cases}\frac{n m^{3}}{4}(2 n-1)+\frac{n m^{2}}{6}(n-1)(n+4) & \text { mis even } \\ +\frac{n m}{6}\left(2 n^{2}-3 n+1\right)+\frac{n-1}{12}\left(2 n^{2}-4 n+3\right), & \\ \frac{n m^{3}}{4}(2 n-1)+\frac{5 m^{2}}{2}\left(\begin{array}{c}n+1 \\ 3\end{array}\right)+\frac{n m}{6}\left(5 n^{2}-9 n+4\right) \quad \text { mis odd } \\ +\frac{1}{12}\left(5 n^{3}-12 n^{2}+10 n-3\right) .\end{cases}$

\section{ACKNOWLEDGEMENT}

The author would like to thank the referee for helpful comments and valuable suggestions.

\section{REFERENCES}

[1] A. R. Ashrafi and M. Mirzargar, "The study of an infinite class of dendrimer nanostars by topological index approaches." J. Appl. Math. Comput., vol. 31, no. 1-2, pp. 289-294, 2009, doi: 10.1007/s12190-008-0211-8.

[2] M. Azari, "Sharp lower bounds on the Narumi-Katayama index of graph operations." Appl. Math. Comput., vol. 239, pp. 409-421, 2014, doi: 10.1016/j.amc.2014.04.088.

[3] M. Azari and A. Iranmanesh, "Chemical graphs constructed from rooted product and their Zagreb indices." MATCH Commun. Math. Comput. Chem., vol. 70, no. 3, pp. 901-919, 2013.

[4] M. Azari and A. Iranmanesh, "Computing the eccentric-distance sum for graph operations." Discrete Appl. Math., vol. 161, no. 18, pp. 2827-2840, 2013, doi: 10.1016/j.dam.2013.06.003.

[5] M. Azari and A. Iranmanesh, "Clusters and various versions of Wiener-type invariants." Kragujevac J. Math., vol. 39, no. 2, pp. 155-171, 2015, doi: 10.5937/KgJMath1502155A. 
[6] M. V. Diudea, QSPR/QSAR Studies by Molecular Descriptors. New York: Nova, 2001.

[7] H. Dong, B. Zhou, and N. Trinajstić, "A novel version of the edge-Szeged index." Croat. Chem. Acta, vol. 84, no. 4, pp. 543-545, 2011, doi: $10.5562 /$ cca1889.

[8] T. Došlić, "Vertex-weighted Wiener polynomials for composite graphs." Ars Math. Contemp., vol. 1, no. 1, pp. 66-80, 2008, doi: 10.7494/opmath.2016.36.1.5.

[9] M. Faghani and A. R. Ashrafi, "Revised and edge revised Szeged indices of graphs." Ars Math. Contemp., vol. 7, no. 1, pp. 153-160, 2014.

[10] G. H. Fath-Tabar, T. Došlić, and A. R. Ashrafi, "On the Szeged and the Laplacian Szeged spectrum of a graph." Linear Algebra Appl., vol. 433, no. 3, pp. 662-671, 2010, doi: 10.1016/j.1aa.2010.03.039.

[11] I. Gutman, "A formula for the Wiener number of trees and its extension to graphs containing cycles." Graph Theory Notes N. Y., vol. 27, no. 9, pp. 9-15, 1994.

[12] I. Gutman and A. R. Ashrafi, "The edge version of the Szeged index." Croat. Chem. Acta, vol. 81, no. 2, pp. 263-266, 2008.

[13] A. Iranmanesh and M. Azari, "Edge-Wiener descriptors in chemical graph theory: A survey." Curr. Org. Chem., vol. 19, no. 3, pp. 219-239, 2015, doi: 10.2174/1385272819666141216225726.

[14] A. Iranmanesh, Y. Pakravesh, and A. Mahmiani, "PI index and edge-Szeged index of HC5C7[k,p] nanotubes." Util. Math., vol. 77, pp. 65-78, 2008.

[15] P. V. Khadikar, “On a novel structural descriptor PI.” Natl. Acad. Sci. Lett., vol. 23, no. 7-8, pp. 113-118, 2000.

[16] P. V. Khadikar, S. Karmarkar, V. K. Agrawal, J. Singh, A. Shrivastava, I. Lukovits, and M. V. Diudea, "Szeged index-Applications for drug modeling." Lett. Drug Design Disc., vol. 2, no. 8, pp. 606-624, 2005, doi: 10.2174/157018005774717334.

[17] M. H. Khalifeh, H. Yousefi-Azari, and A. R. Ashrafi, "Vertex and edge PI indices of Cartesian product graphs." Discrete Appl. Math., vol. 156, no. 10, pp. 1780-1789, 2008, doi: 10.1016/j.dam.2007.08.041.

[18] M. H. Khalifeh, H. Yousefi-Azari, A. R. Ashrafi, and I. Gutman, "The edge Szeged index of product graphs." Croat. Chem. Acta, vol. 81, no. 2, pp. 277-281, 2008.

[19] M. Randić, "On generalization of Wiener index for cyclic structures." Acta Chim. Slov., vol. 49, no. 3, pp. 483-496, 2002, doi: 10.1.1.119.2208.

[20] N. Trinajstić, Chemical Graph Theory. Boca Raton: CRC Press, 1992.

[21] H. Wiener, "Structural determination of paraffin boiling points." J. Am. Chem. Soc., vol. 69, no. 1, pp. 17-20, 1947, doi: 10.1021/ja01193a005.

[22] Y. N. Yeh and I. Gutman, "On the sum of all distances in composite graphs." Discrete Math., vol. 135, no. 1-3, pp. 359-365, 1994, doi: 10.1016/0012-365X(93)E0092-I.

Author's address

Mahdieh Azari

Department of Mathematics, Kazerun Branch, Islamic Azad University, P. O. Box: 73135-168, Kazerun, Iran

E-mail address: azari@kau.ac.ir, mahdie.azari@gmail.com 